\title{
Leveraging Interprofessional Education to Improve Physician/ Laboratory Cooperation and Patient Outcomes
}

\author{
Benjamin W. Weber ${ }^{1} \cdot$ Kamran Mirza ${ }^{2}$ \\ Accepted: 8 December 2021 / Published online: 14 January 2022 \\ (c) The Author(s) under exclusive licence to International Association of Medical Science Educators 2021
}

\begin{abstract}
Pathology and laboratory medicine are integral parts of the healthcare team. While this fact is uncontested, efforts to integrate medical laboratory science (MLS) students into interprofessional education (IPE) activities with medical students have been scarce. We contend here that now is the optimal time to explore IPE with medical and MLS students and that doing so will provide significant benefits to students as well as their future patients. Neglecting to capitalize on this opportunity will result in continued interprofessional barriers.
\end{abstract}

Keywords Healthcare education · Interprofessional education $\cdot$ Medical laboratory science $\cdot$ Undergraduate medical education

The coronavirus disease 2019 (COVID-19) pandemic has changed the healthcare landscape, in both service delivery and education. While devastating vulnerabilities ranging from supply chain management to clinical education site placement were revealed in both domains, opportunities for growth and improvement were also created. A 2020 report published by Centers for Disease Control (CDC) claims that COVID-19 has made the field of public health more visible, and the current global situation sets the ideal stage to recruit, train, and retain professionals in their field [1]. Moreover, the report posited that interprofessional education (IPE) and well-developed knowledge of the roles of other healthcare professionals will improve public health outcomes during disaster and pandemic management; we contend that this concept is easily applicable to other, less acute situations, such as antibiotic resistance monitoring programs, where knowledge of both the clinical aspects of infection care/ control and technical aspects of treatment resistance assays is becoming more relevant as resistances become more complex, require new testing techniques, and grow more dangerous.

Benjamin W. Weber

Bwweber2@wisc.edu

1 School of Medicine and Public Health, University of Wisconsin, Madison, USA

2 Loyola University Chicago Stritch School of Medicine, Maywood, USA
The World Health Organization defines IPE as occurring when "two or more professions learn about, from, and with each other to enable effective collaboration and improve health outcomes," and further describes that the term "professional" should be interpreted to include those who can improve the physical, mental, or social health of a community [2]. We interpret this definition broadly to allow the inclusion of groups that are often neglected from clinically focused IPE, including laboratory, legal, and information technology professionals, as well as community leaders. Interprofessional teams trained in this way will be better able to respond to the needs of the community in which they are embedded [1]. Arguably, pathology and laboratory medicine are at the heart of this interprofessional team-these fields have the power and expertise to affect patient care across time (from sample collection to processing to return of results) and across disciplines such as nursing, pharmacy, and medicine, as well as engage with community leaders to address concerns such as testing availability (as was apparent at the height of the COVID-19 crisis).

Much like public health, we argue that medical laboratory science (MLS) has experienced a dramatic increase in public awareness. We also believe that the same opportunities outlined by the 2020 CDC report apply to the fields of pathology and MLS. COVID-19 has highlighted the need for IPE and has created an environment in which students are comfortable with virtual learning environments-this is ideal for physician/laboratory IPE as, in the future, students will 
likely communicate in ways other than face to face [3]. Additionally, the authors "surmise that within the context of the COVID-19 pandemic, students were able to heighten their connections to the global interprofessional community [3]." IPE activities have the potential to continue to capitalize on such an atmosphere and further develop the sense that, while individuals have different roles within the healthcare team, knowledge of the abilities of others and limitations of one's own profession are required for excellent patient care.

It is imperative that the pathology and MLS community take advantage of this recent development of a more connected interprofessional community. Literature shows a lack of awareness of pathology as a career choice for medical students [4]; IPE with medical students and MLS students may give medical students much needed exposure to laboratory sciences and may improve their outlook on the pathology pathway. A scoping review from 2018 found only two instances of IPE simulation activities that included MLS students; only one of these instances included both MLS and medical students [5-7]. This paucity of inclusion of MLS students in IPE is dramatically apparent and perhaps most startling when one considers that IPE between MLS and medical students has the potential to break down academic and clinical siloing [8]. It has been demonstrated that the role of medical laboratory scientists is poorly understood in some other healthcare fields [9], and authors have claimed that there is a dearth of knowledge about the medical laboratory and lack of exposure to how MLS can assist in the administration and coordination of clinical care [10].

IPE has the potential to not only educate medical students and other health professional students about the roles and responsibilities of MLS students, but also empower MLS students to flex their expertise to improve utilization management, potentially saving costs and improving patient outcomes. This not only is perhaps most easily applied in antibiotic stewardship and resistance monitoring programs, as we described above, but also has opportunities in molecular diagnostics (recommending reflex testing or discussing panel versus single target assays) and in testing related to clotting disorders. Other work has demonstrated the IPE not only improves interprofessional education, but also allows for "enhanced decision making" to emerge [11]; students, while accepting the benefits of healthcare specialization, were more apt to also recognize the limitations of siloing knowledge and were more likely to engage in a collaborative model. While the authors who originally noted the presence of enhanced decision making in the setting of interprofessional practice did not provide specific examples, we envision scenarios including testing selection or exclusion based on local testing panel versus testing send out (including factors such as time to results in the analysis of utility) and selection of the type of antimicrobial resistance testing as excellent demonstrations of enhanced decision making between clinicians and laboratory professionals. Implementing this kind of enhanced decision making with laboratory professionals can only be achieved when ordering providers can effectively work with MLS professionals and understand how to leverage their expertise; laboratory professionals, in turn, must be comfortable approaching ordering providers when they believe that they have critical information that may improve testing strategy, inform specimen collection technique, or bear relevance to some other aspect of patient care.

While data suggest that MLS students are ready for IPE and there is a substantial argument for IPE involving MLS and medical students, there has been data to suggest that MLS students feel less valued by other health professionals after IPE activities [7, 12]. Such data is alarming, and future IPE activities must be designed to validate and empower all professions that participate. Experiences with other professional students demonstrate that early IPE activities may improve student cooperation [13]. Such early encounters, when students are still unsure of their own role in patient care, may be most beneficial when designing MLS/medical student IPE modules; in the pre-clinical years of their education, medical students may be less likely to think of laboratory services as "ancillary"; they may be more willing to accept the integral role laboratory scientists play in patient care. MLS students, in turn, may be more likely to develop improved longitudinal confidence when interacting with patient care professionals. Cases involving antimicrobial resistance, massive blood transfusion, and molecular testing not only are relevant for medical students in their pre-clinical training, but also provide MLS students with a platform to demonstrate how much value they can add to the clinical course of patients in terms of diagnosis, safety, and time to effective treatment.

\section{Declarations}

Ethical Approval NA.

Informed Consent NA

Conflict of Interest The authors declare no competing interests.

\section{References}

1. Brisolara KF, Smith DG. Preparing students for a more public health-aware market in response to COVID-19. Prev Chronic Dis. 2020;17:200251. https://doi.org/10.5888/pcd17.200251

2. World Health Organization. Framework for action on interprofessional education and collaborative practice. World Health Organization. 2010. https://apps.who.int/iris/handle/10665/70185

3. Jones TA, Vidal G, Taylor C. Interprofessional education during the COVID-19 pandemic: finding the good in a bad situation. J Interprof Care [Internet]. 2020;34(5):633-46. Available from: https://doi.org/10.1080/13561820.2020.1801614 
4. Hung T, Jarvis-Selinger S, Ford JC. Residency choices by graduating medical students: why not pathology? Hum Pathol [Internet]. 2011;42(6):802-7. Available from: https://doi.org/10.1016/j. humpath.2010.10.018

5. Lee CA, Pais K, Kelling S, Anderson OS. A scoping review to understand simulation used in interprofessional education. J Interprofessional Educ Pract [Internet]. 2018;13(May):15-23. Available from: https://doi.org/10.1016/j.xjep.2018.08.003

6. King S, Greidanus E, Carbonaro M, Drummond J, Patterson S. Merging social networking environments and formal learning environments to support and facilitate interprofessional instruction. Med Educ Online. 2009;14:5.

7. Beard TS, Robertson TM, Semler JR, Cude CJ. A study of interprofessional collaboration in undergraduate medical laboratory science and nursing education. Am Soc Clin Lab Sci. 2015;28(2):83-90.

8. Hodgkins SR. Interprofessional education and practice, what does this mean for the medical laboratory scientist?. Poster presented at the national meeting of the American Society for Clinical Laboratory Science, Philadelphia, PA. 2016.

9. Rhees JR, Scheese CH, Ward D, Hollister L, Blamires TL, Wilkins DG, et al. Clinical practice simulation for blood transfusion reactions: an interprofessional approach. Am Soc Clin Lab Sci. 2015;28(4):224-31.

10. Ractice CIP. Clinical Laboratory Educators' Conference 2015 Abstracts. Am Soc Clin Lab Sci. 2015;28(2):106-12.

11. Lucas C, Power T, Hayes C, Ferguson C. Two heads are better than one- pharmacy and nursing students perspectives on interprofessional collaboration utilizing the RIPE model of learning. Res Soc Adm Pharm [Internet]. 2020;16(1):25-32. Available from: https://doi.org/10.1016/j.sapharm.2019.01.019

12. Al-Qahtani MF. Measuring healthcare students' attitudes toward interprofessional education. J Taibah Univ Med Sci [Internet]. 2016;11(6):579-85. Available from: https://doi.org/10.1016/j. jtumed.2016.09.003

13. Krause DA, Hollman JH, Pawlina W, Newcomer KL. Interprofessional education: collaboration or competition? A tale of two experiences. Curr Sports Med Rep. 2014;13(5):291-2.

Publisher's Note Springer Nature remains neutral with regard to jurisdictional claims in published maps and institutional affiliations. 